\title{
AUTHOR INDEX (Volume 15)
}

A

Allampallewar, S. B. \& Srividya, A., Corrosion Initiation Time for Reinforced Concrete Members Along Indian Coasts: Effects of Temperature and Relative Humidity - A Probabilistic Approach

Allampallewar, S. B., see Srividya, A.

Atta, A. M. A., see Khoo, M. B. C.

\section{B}

Basirzadeh, M., see Kapur, P. K.

Baskin, E. M., The New Approach for Regression Models Analysis

Bhatkar, M. V., Verma, A. K. \& Karki, N. R., Impact of Level of Contingencies on Wellbeing States of Generating System - A Probabilistic and Fuzzy Approach

Bullard, L. A., see Khoshgoftaar, T. M.

C

Castagliola, P., Celano, G., Fichera, S. \& Nunnari, V., A Variable Sample Size $S^{2}$-EWMA Control Chart for Monitoring the Process Variance

Celano, G., Costa, A., Fichera, S. \& Trovato, E., One-Sided Bayesian $S^{2}$ Control Charts for the Control of Process Dispersion in Finite Production Runs

Celano, G., see Castagliola, P.

Chen, H.-H., see Tsai, T.-R.

Chien, W.-T. K. \& Yang, S. F., Using Reverse Arrangement Test to Detect Non-Monotonic Trends for Semi-Conductor Manufacturing and Reliability Tests

Cho, B. R., see Jeong, H.

Costa, A., see Celano, G.
2 (2008) 113

4 (2008) 367

2 (2008) 101

3 (2008) 247

3 (2008) 181

4 (2008) 305

3 (2008) 181

3 (2008) 203

6 (2008) 497

5 (2008) 441

4 (2008) 305
D

Dandotiya, R., see Kumar, S. $\quad 2$ (2008) 167

Ding, Y., see Li, W. 5 (2008) 425

Dohi, T., see Ishii, T. $\quad 1$ (2008) 1

Dohi, T., see Okamura, H. 1 (2008) 19

Donovan, J., see Joyce, T. 6 (2008) 555

F

Fengqiang, L., see Zhiqiang, S. 5 (2008) 465

Fichera, S., see Castagliola, P. 3 (2008) 181

Fichera, S., see Celano, G. 4 (2008) 305

Fujiwara, T., see Ishii, T. $\quad 1$ (2008) 1

G

Gaffney, O., see Joyce, T.

6 (2008) 555

Gao, K., see Khoshgoftaar, T. M.

Gedela, R. K., see Mohan, K. K.

Goyal, N. K., see Kumar, K. S. 2 (2008) 143

Greco, R., see Marano, G. C. 4 (2008) 391

Gupta, A., Kapur, R. \& Jha, P. C., Considering Testing Efficiency and Testing Resource Consumption Variations in Estimating Software Reliability

2 (2008) 77

\section{$\mathbf{H}$}

Honari, B., see Joyce, T.

6 (2008) 555

Hongwei, X., see Zhiqiang, S. 5 (2008) 465

Hoskins, G., see Putcha, C. S. $\quad 2$ (2008) 159

I

Ishii, T., Fujiwara, T. \& Dohi, T., Bivariate Extension of Software Reliability Modeling with Number of Test Cases

$1(2008) 1$

J

Jeong, H., Song, S., Shin, S. \& Cho, B. R., Integrating Data Mining to a Process Design Using the Robust Bayesian Approach

Jha, P. C., see Gupta, A.

5 (2008) 441

2 (2008) 77

Joyce, T., Honari, B., Wilson, S., Donovan, J. \& Gaffney, O., Models for Optimization of Production Environmental Stress Testing on Electronic Circuit Packs 
K

Kalia, P., see Putcha, C. S.

Kamdar, K. J., see Putcha, C. S.

Kapur, P. K., Khatri, S. K. \& Basirzadeh, M., Software Reliability Assessment Using Artificial Neural Network Based Flexible Model Incorporating Faults of Different Complexity

Kapur, R., see Gupta, A.

Karki, N. R., see Bhatkar, M. V.

Khatri, S. K., see Kapur, P. K.

Khoo, M. B. C., Wu, Z. \& Atta, A. M. A., A Synthetic Control Chart for Monitoring the Process Mean of Skewed Populations Based on the Weighted Variance Method

Khoshgoftaar, T. M., Bullard, L. A. \& Gao, K., A RuleBased Software Quality Classification Model

Kimura, M., Bootstrap Confidence Intervals of Software Reliability Measures Based on a Gamma Function Model

Kumar, K. S., Misra, R. B. \& Goyal, N. K., Development of Fuzzy Software Operational Profile

Kumar, R., see Kumar, S.

Kumar, S., Dandotiya, R., Kumar, R. \& Kumar, U., Inspection Frequency Optimization Model for Degrading Flowlines on an Offshore Platform

Kumar, U., see Kumar, S.

L

Li, W., Zuo, M. J. \& Ding, Y., Optimal Design of Binary Weighted K-Out-of-n Systems

Li, Z., see Liao, H.

Liao, H. \& Li, Z., Multiobjective Design of Equivalent Accelerated Life Testing Plans

Lu, W., see Tsai, T.-R.
2 (2008) 159

2 (2008) 159

2 (2008) 113

2 (2008) 77

2 (2008) 101

2 (2008) 113

3 (2008) 217

3 (2008) 247

1 (2008) 55

6 (2008) 581

2 (2008) 167

2 (2008) 167

2 (2008) 167

5 (2008) 425

6 (2008) 515

6 (2008) 515

3 (2008) 203
M

Maja, R., see Zio, E.

Marano, G. C. \& Greco, R., Performance Reliability Based Optimization Criterion for Elastic Structures Subject to Random Loads Marchionni, G., see Zio, E. Misra, R. B., see Kumar, K. S. Mohan, K. K., Srividya, A. \& Gedela, R. K., Quality of Service Prediction Using Fuzzy Logic and RUP Implementation for Process Oriented Development

$\mathbf{N}$

Naikan, V. N. A., see Syamsundar, A.

Newton, C., see Putcha, C. S

Nunnari, V., see Castagliola, P.

O

Okamura, H. \& Dohi, T., Software Reliability Modeling Based on Mixed Poisson Distributions

$\mathbf{P}$

Páleš, D., see Sadovský, Z.

Pizzano, F., see Putcha, C. S.

Putcha, C. S., Kalia, P., Pizzano, F., Hoskins, G., Newton, C. \& Kamdar, K. J., $A$ Case Study on FMEA Applications to System Reliability Studies

$\mathbf{R}$

Ram, M. \& Singh, S. B., Availability and Cost Analysis of a Parallel Redundant Complex System with Two Types of Failure Under Preemptive-Resume Repair Discipline Using GumbelHougaard Family Copula in Repair

$\mathrm{S}$

Sadovský, Z. \& Páleš, D., Probabilistic Optimization of Partial Safety Factors
$2(2008) 143$

3 (2008) 261

2 (2008) 159

3 (2008) 181

1 (2008) 19

5 (2008) 411

2 (2008) 159

4 (2008) 341

$4(2008) 391$

1 (2008) 67

6 (2008) 581

2 (2008) 159 
for the Design of Industrial Buildings

Sansavini, G., see Zio, E.

Schneidewind, N., ComplexityDriven Reliability Model

Shin, S., see Jeong, H.

Singh, S. B., see Ram, M.

Song, S., see Jeong, H.

Srividya, A. \& Allampallewar, S. B., Corrosion Initiation Time of Reinforced Concrete Structures in Marine Environment - A Probabilistic Approach

Srividya, A., see Allampallewar, S. B.

Srividya, A., see Mohan, K. K.

Syamsundar, A. \& Naikan, V. N. A., Hierarchical Segmented Point Process Models with Multiple Change Points for Maintained Systems

$\mathbf{T}$

Tamura, Y. \& Yamada, S., A Component-Oriented Reliability Assessment Method for Open Source Software

Trovato, E., see Celano, G.

Tsai, T.-R., Bayesian Sampling Plans with Progressive Censoring and Warranty Policy

Tsai, T.-R., Chen, H.-H. \& $\mathrm{Lu}, \mathrm{W} ., \quad$ The Inspection of Acceptance Sampling for Step-Stress Tests with an Equally-Spaced Interval Censoring Scheme

5 (2008) 411

1 (2008) 67

5 (2008) 479

5 (2008) 441

4 (2008) 341

5 (2008) 441

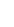

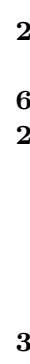

6 (2008) 539

2 (2008) 143
1 (2008) 33

4 (2008) 305

4 (2008) 329

3 (2008) 203

\section{V}

Verma, A. K., see Bhatkar, M. V.

2 (2008) 101

Verma, A. K. \& Vijay Venu, V., Adequacy-Based Power System Reliability Studies in the Deregulated Environment

2 (2008) 129

Vijay Venu, V., see Verma, A. K.

2 (2008) 129

\section{W}

Wilson, S., see Joyce, T. 6 (2008) 555

Wu, Z., see Khoo, M. B. C. 3 (2008) 217

$\mathbf{X}$

Xiujian, S., see Zhiqiang, S. 5 (2008) 465

$\mathbf{Y}$

Yamada, S., see Tamura, Y. 1 (2008) 33

Yang, S. F., see Chien, W.-T. K.

6 (2008) 497

\section{Z}

Zhengyi, L., see Zhiqiang, S.

Zhiqiang, S., Hongwei, X., Xiujian, S., Fengqiang, L. \& Zhengyi, L., An Approach for Human Error Modes and Effects Analysis Using $H T A+E T A$

Zio, E., Sansavini, G., Maja, R. \& Marchionni, G., An Analytical Approach to the Safety of Road Networks $\quad 1$ (2008) 67

Zuo, M. J., see Li, W. $\quad 5$ (2008) 425
5 (2008) 465

5 (2008) 465 